\title{
DVB-T2 technology improvements challenge current strategic planning of ubiquitous media networks
}

\author{
Aleksandar Sugaris ${ }^{1 *}$ and Irini Reljin ${ }^{2}$
}

\begin{abstract}
This article presents an analysis of the key technology improvements of the second generation of Digital Video Broadcasting - Terrestrial (DVB-T2) over its first generation (DVB-T) and an estimation method of the new technology influence on the broadcasting business case used in digital terrestrial networks. This article addresses the current challenges in broadcasting wireless network design and strategic planning of ubiquitous media by taking the multidisciplinary approach. The model presented supports decision-making process driven by an optimal investment cost and the techno-economic analysis of digital terrestrial wireless technologies using innovation service diffusion Bass model. The method of estimating the benefits of advanced digital dividend exploits the capacity gained due to DVB-T2 technology novelties. The simulation results for Serbia show that the profitability of broadcast network is achieved in some cases already after 2 years if DVB-T2 standard is used.
\end{abstract}

Keywords: DVB-T/T2, technology improvement, techno-economic efficiency, PayTV, Bass diffusion model, Serbia

\section{Introduction}

There are several standards currently used worldwide for digital terrestrial broadcasting [1]. The first introduced was the Advanced Television Systems Committee (ATSC), now used in North America and South Korea. Digital Video Broadcasting - Terrestrial (DVB-T) is the European-based consortium standard, used apart from Europe in Australia, India, and some other Asian and African countries. The Japanese standard for digital terrestrial television is called Integrated Services Digital Broadcasting - Terrestrial (ISDB-T), adopted after ATSC and DVB-T taking into account the experience gained with the older standards and it is widely used in South America. Besides those three international standards for digital terrestrial broadcasting, there is also Chinese Digital Terrestrial Multimedia Broadcast standard for fixed and mobile users. Two significant standards for terrestrial mobile TV reception are Terrestrial - Digital Multimedia Broadcasting (T-DMB) and Digital Video Broadcasting - Handheld (DVB-H). The first one was developed in South Korea and its physical parameters

\footnotetext{
*Correspondence: aleksandar_sugaris@hotmail.com

${ }^{1}$ ICT College of Applied Sciences, Zdravka Celara 16, 11000 Belgrade, Serbia Full list of author information is available at the end of the article
}

are identical to the European Digital Audio Broadcasting (DAB) standard. T-DMB is being put into use in many countries around the world. On the other side, DVB-H is the preferred technology for terrestrial mobile broadcasting services in Europe.

The focus of this article is the performance analysis and efficiency estimation method applied to the technologies for fixed reception used in our region - Europe. The new technologies, like compression standard Moving Picture Experts Group 4 (MPEG-4) part 10, or H.264/AVC (hereinafter called MPEG-4) and the second generation of digital terrestrial broadcasting standard (DVB-T2), provide increased capacity and ruggedness in the terrestrial transmission environment. As we indicated in our previous research [2], when considering the whole picture, it is evident that the latest technologies besides technical benefits, increased capacity for new services and higher quality of service, bring also a higher system cost. The viability of digital broadcasting business case is directly influenced by this issue and could make or break the project. We conducted a research with the similar idea as in [3], where broadband wireless access efficiency and economic analysis for licensed TV bands were developed, and as in [4], where the 
profitability evaluation of high-altitude platforms was performed for broadband multimedia services in Korea.

Prior efforts to determine the relationship between technical and economical performance of digital broadcasting technologies, to the best of authors' knowledge, have been rare and different in scope. We believe that is especially the case with DVB-T2.

Since the early days of digital terrestrial broadcasting there were researches [5] toward techno-economic analysis which models market prospects, convergence (Telco, IT, broadcasting), and its regulatory implications. The analysis [5] explores technological issues and drivers in the development of digital terrestrial TV in Denmark. On the other hand, the analysis [6] of the economics of IP datacast service to mobile users over terrestrial DVB system offers techno-economic simulation model which builds business case for a greenfield operator. This model calculates the cost of building and operating IP datacast DVB-H network. The article [7] focuses on DVB-H and DAB standards comparison by analyzing radio and mobile TV services in Sweden in terms of capacity demand and network cost. For that scenario, profit/loss simulation model was not developed. In the analysis [8], we can find techno-economic planning considerations for digital TV systems. The saidarticle proposes generic growth equation for HDTV, IPTV, and mobile TV services. The equation defines driving force (propelling factor) in terms of market penetration growth in \% per annum giving $S$ curve for growth. It is related with S-curve of technological diffusion that we use in this article as one part of our simulation model; however, we use Bass Diffusion Model which will be explained later. The comprehensive study [9], compiled by Analysys Mason, DotEcon and Hogan\&Hartson for the European Commission regarding exploiting the digital dividend, touches apparently very similar topics as we do in this article and our previous research [2], still this is true only at first sight. Even though there are similarities, such as that the study generally estimates economic benefits for different technology profiles (MPEG-2/ DVB-T, MPEG-4/DVB-T, MPEG-4/DVBT2), there are a lot of differences, for instance the estimated economic benefit is taken as the value that potential uses of the digital dividend could generate as the sum of consumer and producer surplus - not as profit/loss break-even analysis, which makes study [9] and our research not directly comparable. First, the approach taken in [9] estimates general welfare of the good to the society instead of estimating the return on investment (ROI) to the investor. Second, we can find in the report [9] that the estimation is based on incremental value generated by digital dividend (value generated by service in $470-862 \mathrm{MHz}$ band above the value that would be realized if the service were only provided by alternate means - different frequency band or telecom network), we calculate incremental benefit (profit) only within the same band. Besides that, one major difference is that in our study we directly compare technical and economical performance of different technology profiles ("head-to-head") for one or multiple multiplexes gained by efficiency difference of different profiles, while the analysis [9] calculates economic value for different technical and demand scenarios for digital dividend gained by switchover from analog-to-digital TV without comparing directly the efficiency of digital technology profiles. Furthermore, the study [9] fills the digital dividend band with a mixture of services and analyzes the value for the whole EU, while we take one service at a time to fill the digital dividend (data or PayTV) for one particular country. On the whole, there is a different purpose of the study [9] than our approach; they analyzed the issues for the purpose of running EU policy against digital dividend 470-862 $\mathrm{MHz}$, while we conducted technical and ROI analysis for the investor. Because of this and other mentioned scope differences with [9], our research could not be directly compared with it.

Generally, there are four stages in techno-economic studies for network deployment planning: scope, model, evaluate, and refine. This approach is known as the Shewhart cycle and it is extended by Deming [10]. In modern literature, we can learn that the cyclic nature is important as it suggests a gradual refinement of the researched business case [11]. We have taken that approach and this article refines the digital broadcasting techno-economic efficiency simulation model presented in [2].

In this article, we improve our previously developed method [2] of estimating the benefits of digital dividend. After a short analysis of DVB-T2 technology improvements over DVB-T, the article presentsthe model which estimates the break-even point where in time the revenue from the new service brought by MPEG-4/DVBT2 meets the system investment level. We proposed a PayTV service to be used within the gained capacity and to generate revenue. For the purpose of estimating the service acceptance rate, we applied Bass Diffusion Model to PayTV service within DVB-T/T2. Alternatively, the simulation model could combine two revenue components: PayTV service and spectrum auction. The break-even analysis takes into account multiple multiplex scenarios. In this way, the techno-economic analysis of multimedia networks strategic planning is expressed through the model which is applied to the Serbian broadcast market. After the presentation of simulation results, the analysis discussion is performed, followed by appropriate conclusions. 


\section{DVB-T2 technology improvement over DVB-T}

This section provides an overview of major technology improvements used in DVB-T2 against DVB-Ttechniques. Those improvements have great impact on service planning simply by increasing transmission capacity through robustness gain. That gain is achieved using the technologies that we analyzed earlier [12] (supported by appropriate ETSI standard [13] and DVB document [14]).

The DVB-T2 specification implements the latest developments in modulation and error-correction to increase the bit-rate capacityand improve signal robustness compared to already successful DVB-T standard (robust system where the DVB-T signal could be detected with a signal-to-noise ratio of only $-12 \mathrm{~dB}$ by using the signal's second-order statistics [15]). Therefore, the changes needed to be made to the physical layer, in order to optimize the system performance for the propagation characteristics of the frequency selective channel and finally impact network configuration. The DVB-T2 specification (like DVB-T) uses Orthogonal Frequency Division Multiplex (OFDM) modulation. However, the introduction of the 256 QAM mode in the DVB-T2 specification increases the number of bits carried per data cell and with the protection from a new powerful forward error correction (FEC) gives capacity improvement. DVB-T2 specification uses two new FEC techniques, low-density parity-check (LDPC) codes in combination with Bose-Chaudhuri-Hocquengham $(\mathrm{BCH})$, correcting errors that are imposed by difficult channel characteristic. When compared to DVB-T standard, which uses Reed-Solomon and convolutional coding, DVB-T2 introduces two additional code rates. One of the main improvements is that DVB-T2 can ensure choice of various robustness and protection levels in the propagation channel within a transport stream for each service separately, so by using Physical Layer Pipes each service can have a different modulation mode depending on the required signal robustness. Like the DVB-T standard, the DVB-T2 specification uses scattered pilot patterns which help the receiver to compensate for the changes (time and frequency) in channels, but DVB-T2 gives wider choice of scattered pilot patterns (eight) that can be selected depending on the FFT size and guardinterval fraction adopted for the particular transmission. This reduces the pilot overhead while assuring a sufficient channel-estimation quality.

Signal robustness against external influences (e.g., geography, buildings) is significantly improved with DVBT2 specification. This is achieved through the use of the rotated constellations technique and time and frequency interleaving. Rotated constellation is a novel technique which ensures that loss of data from one channel component can be recovered in another channel component. After being created, a QAM constellation is rotated in the complex I-Q plane so that each axis on its own carries enough information to distinguish between the two neighboring points in the constellation. The I and Q components are now separated by the interleaving process so that in general they travel on different frequencies, and at different times. At the receiver plane, the values are recombined to give the original rotated constellation. Time and frequency interleaving additionally improve signal robustness against disturbances such as time impulsive noise and frequency selective response.

With DVB-T2 we can implement larger Single Frequency Network (SFN) than with DVB-T. New carrier modes (FFT size) in DVB-T2 increase the symbol period so we can implement longer distances between SFN transmitters. This increase in the symbol period can still handle multipath reflections if we reduce proportional size of the guard interval. It is possible to do so as DVB-T2 provides wider choice for guard interval fraction which gives greater flexibility in designing anSFN as well as Multiple Frequency Network. Optionally, DVBT2 can use Alamouti coding mode that is especially effective in SFNs where a receiver can benefit from multiple input signals simultaneously received from more than one transmitter (Multiple Input Single Output MISO).

For the introduction of data service into digital broadcasting system as the access network, it is very important that besides classical encapsulation protocol MPEG TS for DVB services, DVB-T2 provides the Generic Stream Encapsulation (GSE) designed to provide appropriate packetization for IP traffic.

The most frequently used method to carry IP datagrams over MPEG TS is Multiprotocol Encapsulation (MPE); however, MPEG TS is a protocol optimized for broadcasting and not for IP services, so MPE suffers from design constrains as DVB protocol use MPEG TS at the link layer. If we consider the channel bandwidth efficiency, the encapsulation of IP datagrams by the MPEG TS MPE adds additional overheads to the transmitted data, thus reducing efficiency compared to GSE that is designed to carry general data, mainly over IP protocol. GSE efficiently encapsulates IP datagrams over variable length link layer packets, which are then directly inserted to the physical layer baseband frames. If IP datagrams use GSE, the reduction in overhead is more than two times compared to MPEG-TS transmission.

Table 1 gives an overview of differences between DVB-T and DVB-T2 digital terrestrial broadcasting standards. 
Table 1 DVB-T vs. DVB-T2 - features overview

\begin{tabular}{|c|c|c|}
\hline & DVB-T & DVB-T2 \\
\hline FEC & $\begin{array}{l}\text { Conv. Coding + RS } \\
1 / 2,2 / 3,3 / 4,5 / 6,7 / 8 \\
\end{array}$ & $\begin{array}{l}\mathrm{LDPC}+\mathrm{BCH} \\
1 / 2,3 / 5,2 / 3,3 / 4,4 / 5,5 / 6\end{array}$ \\
\hline Modulation & COFDM: QPSK, 16QAM, 64QAM & COFDM: QPSK, 16QAM, 64QAM, 256QAM \\
\hline Guard interval & $1 / 4,1 / 8,1 / 16,1 / 32$ & $1 / 4,19 / 256,1 / 8,19 / 128,1 / 16,1 / 32,1 / 128$ \\
\hline FFT size & $2 k, 8 k$ & $1 k, 2 k, 4 k, 8 k, 16 k, 32 k$ \\
\hline Scattered pilots & $8 \%$ of total & $1 \%, 2 \%, 4 \%, 8 \%$ of total \\
\hline Frequency bands & Band III, IVN (VHF, UHF) & Band III, IVN (VHF, UHF) + L Band \\
\hline Channel raster & $6,7,8 \mathrm{MHz}$ & $1.75,5,6,7,8,10 \mathrm{MHz}$ \\
\hline $\mathrm{C} / \mathrm{N}$ range (Rice channel) & 5 (QPSK 1/2) to 23 dB (64QAM 7/8) & 3(QPSK 1/2) to 24 dB (256QAM 5/6) \\
\hline Max SFN Tx distance $(8 \mathrm{MHz})$ & $67.2 \mathrm{~km}$ (8k mode) & 159.6 km (32k mode), 134.4 km (16k mode) \\
\hline Rotated constellation & - & Robustness gain in channels with multipath, SFN, interference,... \\
\hline Interleaving & Bit, frequency & Bit, cell, time, frequency \\
\hline Diversity & SISO (SIMO if diversity receiver) & $\mathrm{SISO}, \mathrm{MISO}$ (SIMO, MIMO if diversity receiver) \\
\hline Max bit rate & 31.7 Mbps (8k, 64QAM, CR = 7/8, Gl = 1/32) & $50.3 \mathrm{Mbps}$ (32ke, 256QAM, CR = 5/6, Gl $=1 / 128,1 \%$ scattered pilots) \\
\hline Data over DVB protocol & MPEG TS MPE & MPEG TS MPE, GSE \\
\hline
\end{tabular}

It could be estimated that by using the technology improvements overviewed above, a new DVB-T2 digital terrestrial broadcasting standard allows potential capacity gain from 50 up to $90 \%$ compared with a DVB-T mode of similar robustness. Alternatively, the capacity could be maintained the same, but in that case, by using DVB-T2 we can increase the signal robustness and the service coverage area as well. Either way multimedia services become ubiquitous. However, due to their complexity, it is not easy to define precisely the key parameters of DVB-T2 because those parameters are more critical for planning DVB-T2 networks than they are for DVB-T.

The exact capacity gain that can be achieved using the DVB-T2 specification in comparison with the DVB-T standard is dependent on the system parameter set chosen estimating the similar coverage area and the same quality of the service. Based on this condition, we take technology profiles for both standards and from Table 2 we can conclude that capacity gain is around $66 \%$. We used the same parameters in our previous research [2] where we explained the reasons for choosing them and the network architecture (Figure 1).

Table 2 DVB-T versus DVB-T2 - capacity comparison

\begin{tabular}{lll}
\hline & DVB-T & DVB-T2 \\
\hline Modulation & 64QAM & 256QAM \\
\hline Carrier number & $8 \mathrm{~K}$ & $32 \mathrm{~K}$ \\
\hline Guard Interval & $1 / 32$ & $1 / 128$ \\
\hline FEC & $2 / 3 C C+\mathrm{RS}$ & $2 / 3 \mathrm{LDPC}+\mathrm{BCH}$ \\
\hline Carrier mode & Standard & Extended \\
\hline Capacity & $24.1 \mathrm{Mbit} / \mathrm{s}$ & $40.1 \mathrm{Mbit} / \mathrm{s}$ \\
\hline
\end{tabular}

In the addition to the technology DVB-T/T2 analysis, this article evaluates economic efficiency as well, using the simulation model for break-even point estimation (profit/loss analysis) that is important factor in building a business case. Before we explain it, in the next section we will describe how the service diffusion model is implemented into the model of ours.

\section{The Bass diffusion model}

As noted in the "Introduction" section, in this article we anticipate the usage of PayTV service in the capacity gain thatwe obtained, based on the explanation from the previous section. In order to estimate the revenue from PayTV service subscription needed for our business case profit/loss calculations, we have to answer a simple

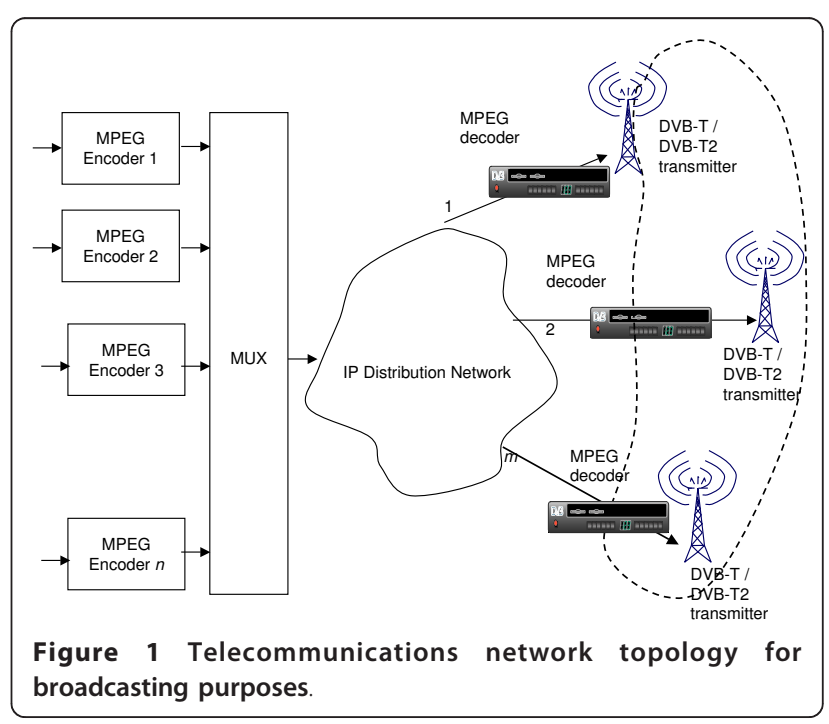


question: what is the service acceptance rate over time for a particular market? The answer is not so simple. This prediction is difficult as no historical data exist for that innovative service on the market (no PayTV over digital terrestrial television network) based on which we could make an estimation of service acceptance rate. Basically, we need to know how to forecast the diffusion of a new service prior to product launch. The theory which helps in that matter is the Diffusion of Innovation established by Rogers [16] and the most prominent mathematical extension to the theory is Bass Diffusion Model introduced in 1969 by Bass [17].

Modeling and forecasting the diffusion of innovations is an important broad research topic. Its importance is justified by the literally thousands of publications regarding it and that field of research is represented by more scholars in more disciplines than many other fields of research. This field is a subject of practical and academic research in disciplines of marketing, engineering, and economy. Forecasting diffusion of innovation in many cases has the strategic importance of new technologies (like DVB-T2) in planning the growth of an economy, but is not less important in helping managers to plan more accurately their ROI strategy, by modeling the development of a service or technology demand (like PayTV). In this article, we use Bass Diffusion Model for that purpose.

The popularity of Bass model could be explained by its applicability to wide variety of diffusion patterns observed in practice. Bass model is very simple as it contains only three parameters, which describe three types of effects: external effects (coefficient of innovation $-p$ ), internal effects (coefficient of imitation - q), and saturation level (total potential market $-m$ ). According to Bass model assumption, there are two channels of communication that influence potential adopters of an innovation, namely mass media $(p)$ and interpersonal channels $(q)$. Individuals adopting a new product because of what they see or hear from the mass media advertising messages occur continually but, it is important, reach the maximum early in the product life cycle. Regarding the interpersonal channels, individuals adopt product based on what they see or hear from prior adopters.

Bass specified the probability of individual adoption in the so-called hazard rate function in below equation

$$
P(t)=\frac{f(t)}{1-F(t)}=p+q \times F(t)
$$

where $f(t)$ is the density function of time to adoption (non-cumulative proportion of the potential market that adopts at time $t$ ) and $F(t)$ is the distribution function of time to adoption (cumulative proportion of the potential market that has adopted up to and including time $t$ ). Simply said, $f(t)$ is the non-cumulative portion that adopt at time $t$, while $F(t)$ is the fraction of the total potential market that has adopted the product at time $t$ and the relationships between them are

$$
\begin{aligned}
& f(t)=\frac{d F(t)}{d t} \\
& F(t)=\int_{i=0}^{t} f(i) d i, \quad F(0)=0
\end{aligned}
$$

If we substitute Equation (2) by Equation (1) and rearrange, we obtain

$$
\frac{d F(t)}{d t}=(p+q \times F(t)) \times(1-F(t))
$$

the differential equation which is besidesEquation (1) another form of the Bass Model. Equation (4) relates a variable $F(t)$ to its derivative of the first-order, $d F(t) / d t$, which is the rate of change of $F(t)$ at time $t$. The solution to the Bass Model differential equation above is

$$
F(t)=\frac{1-e^{-(p+q) \times t}}{1+\frac{q}{p} \times e^{-(p+q) \times t}}
$$

As indicated earlier, the parameter $m$ represents the total market, which is the ultimate number of purchasers of the service over the life of the service. Since $f(t)$ is the likelihood of adoption at $t$ and $F(t)$ is the cumulative proportion of the total potential market that has adopted at time $t$, the number of adopters (first-time buyers) at time $t$, which is sometimes called "sales" at $t$, is

$$
S(t)=m \times f(t)
$$

and the cumulative number of adopters up to and including time $t$ is

$$
Y(t)=m \times F(t)
$$

In the continuous Bass Model, time $t$ can be any nonnegative value and delta $t$ can be extremely small $(d t \rightarrow 0)$. In the real world of sales records, which are kept in different integer time units (usually months or years not continuously), we have to translate continuous time relationships to discrete time intervals, from $(t-1)$ to $t$, that may be numbered $t=0,1,2, \ldots$ which represents months or years. In discrete time models where time can only take values such as $1,2,3, \ldots$ Equations (1) and (5) should not be used together for forecasting product diffusion because they are inconsistent. In order to avoid circular and inconsistent relationships, the 
continuous Bass Model has been modified in different discrete versions even in the original Bass paper [17] (although that version is frequently referenced it introduces substantial error into the model). The discrete Bass Model that is preferred is explained in [18]. In short form, that discrete model is described as

$$
\begin{aligned}
& F(t)=\frac{1-e^{-(p+q) \times t}}{1+\frac{q}{p} \times e^{-(p+q) \times t}} \\
& f(t)=\left\{\begin{array}{cc}
F(t) & \text { if } t=1 \\
F(t)-F(t-1) & \text { if } t>1
\end{array}\right\}
\end{aligned}
$$

In order to forecast the diffusion of the service (service acceptance rate), we need to know three parameters: $p, q$, and $m$. There are three main procedures to estimate the parameters of the diffusion predictive model. Those estimations of $p, q$, and $m$ are from historical sales data, using: Ordinary Least Squares (OLS) linear regression analysis, Nonlinear Least Squares (NLS) optimization, or analogous product estimates of $p$ and $q$. The first two are used commonly when the product/service is already launched and sales record exists. As we do not have PayTV service introduced in our DVB-T/T2 system, we estimate service adoption by using analogous service parameter because no sales data are available. That approach was explained and successfully implemented in [19] where Bass et al. contributed to forecasting diffusion of satellite television.

In the method of guessing by analogy, the first step is to find similar new service that was introduced earlier and where historical data were used for estimating $p$ and $q$ by OLS or NLS method. The second step is to use the coefficients estimated from the diffusion scheme of analogous service. Thus, for $p$ and $q$, the task is to select from the set of previously known introduced services thebest analogy to use. Different industry categories of products and services are listed [20] with $p$ and $q$ data available. The best approach in choosing the analogy service is to use the analogs based on the similarities in expected market reactions rather than the similarities of service itself. Following this approach, in searching for analogies of PayTV service we focused around other television services/products - color TV, terrestrial TV, satellite TV, cable TV and IPTV. Among them we have chosen cable TV as the most similar to PayTV because it is subscription service in its nature, while others could be used simply by pointing the antenna and tuning to the right frequency... with the exception of IPTV. The reason we have not chosen IPTV is because in literature there are not enough reliable data for $p$ and $q$ parameters of Bass model as this is a quite new service (with the history of less than 10 years). Cable TV is listed in [20] with $p=0.100$ and $q=$ 0.060. Analyzing further this analogy in terms of the similarities in expected market reactions, we found one significant difference between selected cable TV service and PayTV. PayTV is the additional service which comes on top of the basic TV programs received by terrestrial digital network as free-to-air content while cable TV subscriber pays subscription fee for the whole package (basic + additional programs) and usually there is no other way that a subscriber home gets TV program. That fact makes PayTV a kind of luxury for the broadcasting market compared to cable TV as elementary TV service. We believe the imitator $q$ parameter will not be changed because it reflects the power of internal influences in the industry which is not varied by the observed fact. The innovator $p$ parameter should be changed because $p$ is usually regarded as being influenced by the firm's advertising policy (impact of mass media) and if we treat PayTV as luxury the impact of advertising cannot be as high as for cable TV because it is not the necessity (the advertising have greater impact to the market for necessity product than for the luxury product). This is the reason why we use in this article $p$ $=0.03$ for PayTV as the average across the studies for the long list of different products and technologies [20]. That value is lower than for cable TV and as $p$ depends on firm's advertising policy which could vary a lot, we believe it makes sense to implement the average value.

On the other hand, if using guessing by analogy it is wise to estimate the Bass Model parameter $m$ (the ultimate number of subscribers) independent of the model. There are different options; estimate can be obtained from analyst forecasts, marketing research, or guessing by testing the logic and assumption behind the estimate (for example using the Delphi Method). If for any reason those options are not used, even though it may be optimistic in many Bass Model implementations, management usually estimates parameter $m$ by having strong intuitive feel about the size of the market. In most cases that judgment is supported by the survey data from consumers on stated intention to subscribe [19]. While related to actual behavior, it is well known that stated intentions overstate actual subscription behavior. We want to estimate the ultimate number of subscribers in Serbia for PayTV service over the digital terrestrial broadcasting network. The estimation starts from the number of households on the market that is for Serbia at the moment 2.5 million. Around one million households are equipped with cable TV and they will probably not convert to digital terrestrial. So, there are around 1.5 million potential TV homes that would receive digital TV program by aerial. In [19], it was successfully predicted that $16 \%$ of TV homes would ultimatelyadopt satellite TV from one particular US satellite broadcast 
operator. Taking this as a reference for our case, it is unrealistically optimistic to assume that $16 \%$ of TV homes in Serbia will adopt PayTV. Taking into account that PayTV is the additional TV programming source and not the total package, our guess is that percentage will be four times lower than in [19] hence ultimately only $4 \%$ of TV homes in Serbia will adopt PayTV over the digital terrestrial broadcasting network. These calculations bring us to the figure of roughly 60,000 subscribers as the saturation level. In this article, we take coefficient $m$ as the parameter and in our simulation model we used two values, 15,000 up and down from the number above ( $m=45,000$ and $m=75,000)$.

In this article, we use the discrete Bass model, Equations (6), (7), and (8), to estimate the monthly increase of PayTV subscriber base and cumulative number of service adopters which is the input variable in our model for simulating total revenue from additional digital dividend and further, the economic efficiency of DVB-T2 technology improvements. For Serbian business case, as explained in this section, we take Bass model coefficients $p=0.030, q=0.060$ with the variation of $m$, $m=45,000$ and $m=75,000$. Figure 2 illustrates the Bass model implementation using the parameter values mentioned above. The estimated sales over time are presented with curves for two ultimate numbers of subscribers, $m$. The third curve represents the real sales over time for IPTV service in Serbia. We obtained the real sales record from our colleagues from Telecom Serbia that have commercially been providing service since 2009. The curve shows the number of new subscribers per month during the first 12 months (2009/10) from the service launch. We want to compare our estimation for PayTV with IPTV real data as: IPTV (with cable TV) has the best similarity to PayTV, we have the real sales

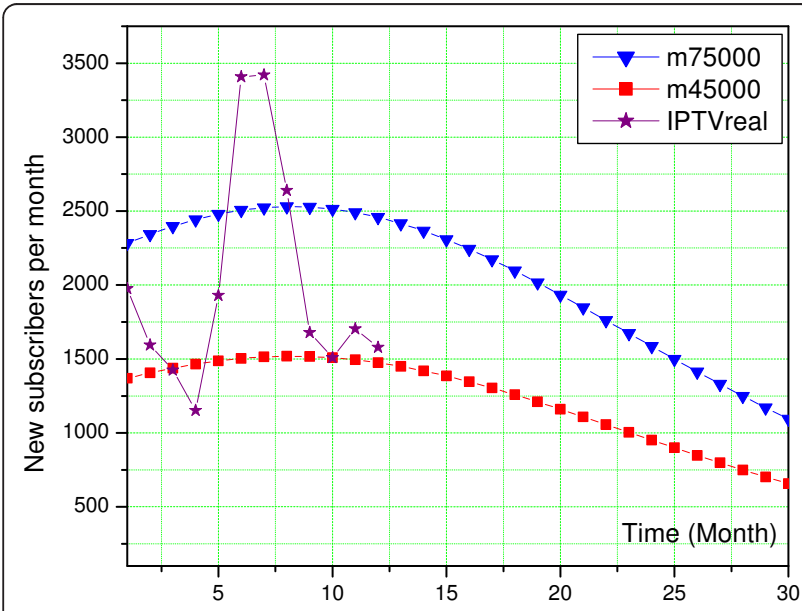

Figure 2 New PayTV subscribers per month in Serbia calculated by Bass Diffusion Model. data of the same (Serbian) market and its introduction was closer to the launch time for PayTV than any other Serbian TV service. From Figure 2, we can see the similar trend for estimated PayTV new subscribers and IPTV real sales, concluding that there is a good match of Bass Model parameters.

\section{Digital broadcasting business case simulation model}

As indicated in the section "Introduction", this article refines the model developed in [2]. The model presented in this study estimates the cost of the MPEG-4/DVB-T2 broadcasting system which uses the latest technologies, calculates the potential revenue from new services brought by new technologies compared to MPEG-4/ DVB-T, and estimates break-even point where the revenue meets the level of invested capital in broadcast infrastructure.

In calculating economic profit $(E P)$, the total cost $(T C)$ is deducted from the total revenue $(T R)$ earned.

$$
E P=T R-T C
$$

$T C$ calculates capital investment in equipment required for the DVB-T2 system and those expenses occur at the beginning of the project implementation. When the general evaluation is needed, the model starts from the idea that $T C$ can be estimated from sales records of the most influential broadcast technology vendors. If the vendor is known for a particular project, then concrete data from the project could be applied to the model for calculating system cost. On the other hand, the revenue $(T R)$ is collected within each time unit from the PayTV service subscription (the revenue from services enabled by new technologies). In this article, we estimate the influence of DVB-T2 technology improvement (over DVB-T) on digital broadcasting business case, so $T R$ calculates the revenue from service gained by capacity improvement and estimates the time when the whole investment would be paid off based only on revenue from technology improvements. When $T R$ is lower than $T C$, the system generates the loss and after break-even point (TR is higher than $T C$ ) the profit is achieved.

As we want to estimate the profit/loss timing for the investment in infrastructure of new technologies (MPEG-4/DVB-T2), in this model we calculate $T C$ as

$$
\text { TC }=\text { HeadEnd }+ \text { Transmitter }
$$

where HeadEnd and Transmitter are the equipment costs at the headend and transmitter sites, which are calculated using the following formula

$$
\text { HeadEnd }=n \times \text { Cencmpg } 4+\text { MUX }
$$




$$
\text { Transmitter }=t \times k \times(C d e c m p g 4+C d v b t 2)
$$

Within Equations (11) and (12) we have introduced the variables:

$n$ - number of TV programs for defined coverage zone; Cencmpg4 - cost of MPEG-4 encoder; MUX - cost of the multiplexer; $t$ - number of transmitter sites; $k$ number of multiplexes; Cdecmpg4 - cost of MPEG-4 decoder; Cdvbt2 - cost of DVB-T2 transmitter.

Except for the variable $k$, all others are independent input variables. Variable $k$ depends on variable $j_{1}$, which is related to transmission mode/profile that gives system useful bitrate $(U B R)$ and to the bit rate of one compressed TV program:

$$
j_{1}=\lfloor U B R d v b t / M P G 4 S D\rfloor
$$

where $U B R d v b t$ refers to useful bit rate for DVB-T equivalent profile (input data from network planners) and MPG4SD is standard definition (SD) TV program MPEG-4 bit rate (input defined by content provider). After dividing, we need to round this ratio to awhole number by just dropping the decimal part. The number of TV programs per one multiplex in DVB-T is represented by $j_{1}$. Now $k$ could be determined by inequality

$$
k \times j_{1} \geq n
$$

as the first whole number from zero that fulfill (14). The number of TV programs per one multiplex in DVB-T2 is represented by $j_{2}$ and is calculated as

$$
j_{2}=\lfloor U B R d v b t 2 / M P G 4 S D\rfloor
$$

where UBRdvbt2 refers to Useful Bit Rate for DVBT2 equivalent profile (input data from network planners).

In this research, we take multiple multiplex scenarios that support broadcasting of $n$ number of TV programs for defined coverage zone. The number of multiplexes and RF channels are defined by variable $k$ - inequality (14), using DVB-T standard. Due to technology improvements introduced in DVB-T2 that are explained earlier in this article, the number of TV programs per one multiplex in DVB-T2 $\left(j_{2}\right)$ is higher than in DVB-T $\left(j_{1}\right)$; so, the required number of multiplexes and RF channels to support $n$ number of TV programs for defined coverage zone is lower for DVB-T2 than DVB-T (it is at least equal). Despite that fact, the model estimates the total cost of the infrastructure $(T C)$ using $k$ number of multiplexes and RF channels in DVB-T2 system, the same number as for DVB-T because we simulate a scenario where the number of RF channels used is the same for both standards as well as the appropriate cost for frequency license. Having that approach in mind, after broadcasting the required number of free-toair TV programs $(n)$ in DVB-T2 there are $\left(k \times j_{2}-n\right)$ and in DVB-T $\left(k \times j_{1}-n\right)$ spare TV programs with which we can broadcast PayTV service. As we want to estimate how fast the investment in DVB-T2 can be returned based on the revenue resulting from technology improvement and less capacity needed for the same number of TV channels we introduce a variable $a$ as advanced digital dividend in number of TV programs, which can be calculated as

$$
\begin{aligned}
& a=\left(k \times j_{2}-n\right)-\left(k \times j_{1}-n\right) \\
& a=k \times\left(j_{2}-j_{1}\right)
\end{aligned}
$$

$T R$ is the revenue gained from the usage of new technologies compared to current technology. If the network operator invest in broadcasting infrastructure, that operator can benefit from the spare capacity $a$ gained by using new technologies. That benefit is the revenue from PayTV service subscription from $a$ TV programs. The cumulative revenue collected after month $M\left(T R_{M}\right)$ is calculated as:

$$
T R_{M}=a \times P \times \sum_{t=1}^{M} S U B_{t}
$$

where $\sum_{t=1}^{M} S U B_{t}$ is the sum of cumulative number of subscribers who pay service through total period from month 1 until month $M$ and variable $P$ is DVB network operator profit from one PayTV program per subscriber per month. $S U B_{t}$ is the cumulative number of subscribers at time $t$ calculated by discrete Bass Model using formulas (7) and (8). $P$ is input variable which is mainly the difference between DVB operator PayTV service price for subscriber per month and the content provider wholesale price for retailer (DVB operator) per subscriber per month. Generally, those prices include cost of condition access solutions on both sides. $P$ varies a lot depending on economic situation in the country, market conditions in the industry, retailer's efficiency, type of the content (Hollywood blockbuster, NBA or Premier League, or something else), whether PayTV is bundled with other services (broadband, telephone) or not, desired profit, etc.

By using this model, total cost is given by (10) while total revenue is given by (17) and with associated formulas we can calculate $T C$ and $T R$ as a function of time and present results on the diagram. From these calculationsand diagram we can easily find the break-even point, practically the period from which the investment becomes profitable. 


\section{The variation of simulation model}

The simulation model presented in the previous section was revised by introducing a new source of revenue. The new source is revenue from auction of the spectrum that is released due to application of the latest broadcasting technologies. In this variation of the model, we maintained the calculation of the total cost (TC) by using the formulas (10), (11), and (12). On the other hand, the total revenue $(T R)$ is calculated by combining the revenue from PayTV service and the revenue from spectrum auction. In this scenario, DVB-T multiplex is fully filled in with TV programs for defined coverage zones. The number of these TV programs $(n)$ is calculated by the formula

$$
n=k \times j_{1}
$$

where $k$ is the input variable (number of multiplexes) and $j_{1}$ (number of TV programs per one multiplex in DVB-T) is calculated by Equation (13). If instead of DVB-T, we apply DVB-T2 standard for the same number of TV programs $(n)$ for defined coverage zones, the required number of multiplexes will be the least integer that is greater than or equal to $n / j_{2}\left(\left\lceil n / j_{2}\right\rceil\right)$. Variable $j_{2}$ (number of TV programs per one multiplex in DVB-T2) is calculated by Equation (15). If by using DVB-T2 system, we implement $k$ multiplexes and RF UHF channels, as we did in the previous section, the digital dividend will consist of two components.

The first component is represented by the whole number $(b)$ of UHF channels $(8 \mathrm{MHz})$ that we calculate as the difference between the implemented number of multiplexes $k$ and the required number of multiplexes for $n$ TV programs if DVB-T2 were used $\left\lceil n / j_{2}\right\rceil$.

$$
b=k-\left\lceil\frac{n}{j_{2}}\right\rceil
$$

The number of UHF channels $b$ brings the total frequency spectrum $(c)$ in $\mathrm{MHz}$

$$
c=b \times 8
$$

The development of mobile broadband access is provided through a number of recommendations and decisions of international and European institutions that develop this area of electronic communications. In many countries, the mobile broadband access is evaluated as the solution for the problem of digital divide. For that reason, some members of the European Union (Germany, Sweden, etc.) have already carried out the procedures of 800 $\mathrm{MHz}$ spectrum auction in the UHF frequency bands (790$862 \mathrm{MHz}$ ), which is in Europe recommended for digital dividend services. That frequency band $(800 \mathrm{MHz})$ attracted the highest attention of mobile communications industry because the propagation characteristics of this sub-1 GHz band are better for long range coverage outside urban areas and better for in-building penetration in dense urban areas. This spectrum could be particularly profitable if it were used by the fourth generation of mobile telecommunication system (3GPP LTE - 3rd Generation Partnership Project Long Term Evolution). Bearing that in mind, in the variation of our model the frequency spectrum $(c)$ in $\mathrm{MHz}$, which is gained by application of the most advanced technologies (MPEG-4/DVB-T2), is used by LTE technology. LTE is designed for scalable channel bandwidths up to $20 \mathrm{MHz}$, with smaller bandwidths covering $1.25,2.5,5$, and $10 \mathrm{MHz}$ for narrow allocations. The channels could be used as paired (Frequency Division Duplex) or unpaired (Time Division Duplex) spectrum. In our model, we use paired channels $2 \times 2.5 \mathrm{MHz}=5 \mathrm{MHz}$ for LTE mobile applications. The number of paired channels, which fit to the spectrum of the whole number of UHF channels as part of first digital dividend component, can be calculated as

$$
d=\left\lfloor\frac{c}{5}\right\rfloor
$$

that brings the available spectrum ( $S$ in $\mathrm{MHz}$ ) for auction

$$
S=d \times 5
$$

The most common measure of price for spectrum is $€ / \mathrm{MHz} /$ head of country's population ( $€ / \mathrm{MHz} / \mathrm{pop})$. There are significant variations in prices across the different bands and across the different countries. The license for spectrum normally expires after 10 to 15 years from auction. We can estimate the appropriate price for spectrum $(P S)$ from the analysis and spectrum value database [21] and this is the input variable.

Finally, we calculate the first component of digital dividend, which comes from revenue of mobile broadband auction (MBA) process, as

$$
M B A=P S \times S \times P O P
$$

where $P O P$ represents the size of country's population. The second component of digital dividend in this variation of the model is revenue from PayTV service according to the formula (17). The question is what the number of PayTV programs $(a)$ is. It is equal to the difference between number of TV programs within the required number of multiplexes for $n$ free-to-air TV programs if DVB-T2 were used $\left(\left\lceil n / j_{2}\right\rceil \times j_{2}\right)$ and the number of TV programs $(n)$ for defined coverage zones.

$$
a=\left\lceil\frac{n}{j_{2}}\right\rceil \times j_{2}-n
$$


Now, the total revenue is calculated as

$$
T R=M B A+T R_{M}=P S \times S \times P O P+a \times P \times \sum_{t=1}^{M} S U B_{t}
$$

where $S$ and $a$ are calculated by formulas (22) and (24), respectively, and $\sum_{t=1}^{M} S U B_{t}$ in the same way as in the previous section.

\section{Simulation results: an application to the Serbian case}

This section estimates the $T C$ and $T R$ of the network operator, following the process described in the previous two sections. For the input variables of the model described, we have taken the values that match to the market in Serbia, but they can apply to some other country with similar size, technical requirements, and economic power.

We will first show the simulation results for the basic model where the revenue is calculated from PayTV service only. By using the input data from network planners summarized in Table $2 U B R d v b t=24.1 \mathrm{Mbps}$ and UBRdvbt2 = 40.1 Mbps (useful bit-rate for DVB-T and DVB-T2 configuration, respectively), input data from content provider MPG4SD = $2.5 \mathrm{Mbps}$ (MPEG-4 bitrate for SD TV), and Equations (13), (14), and (15), we can calculate the number of RF channels/multiplexes necessary for carrying specific number of TV programs using the values of variables highlighted above (Figure $3)$. By reading appropriate values from Figure 3 and using Equation (16) we calculate advanced digital dividend $(a)$ related to number of TV programs for defined coverage zone $(n)$.

Besides the input data from network planners and content providers, cost of the equipment (encoders,

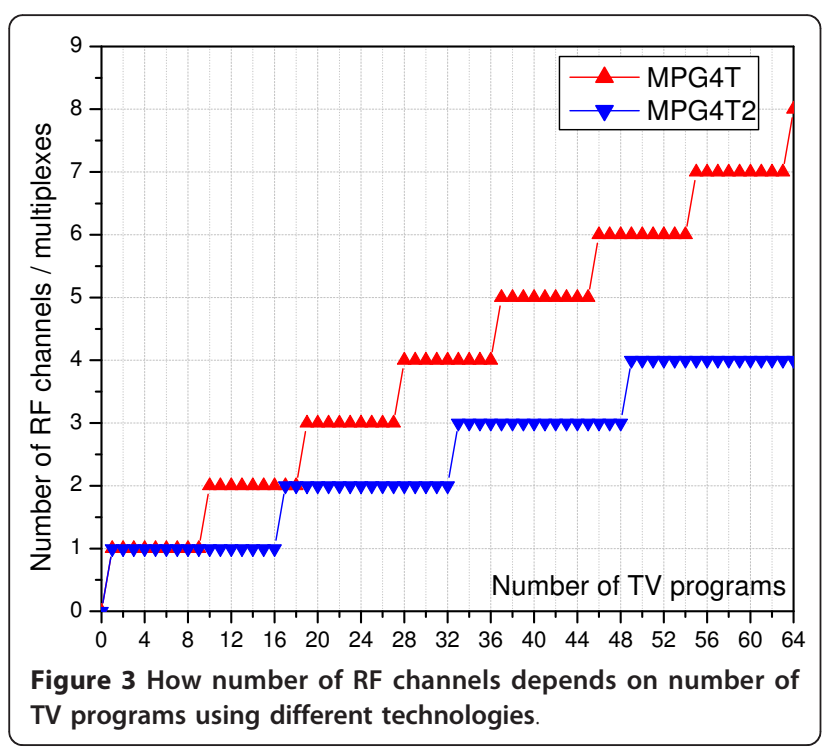

decoders, multiplexers, and transmitters) plays an important role in the model simulation. Like in [2], the authors selected the information source basedon their best knowledge of influential vendors in the broadcasting and networking field and again according to the level of vendor willingness to cooperate on this research. We used sales records for cost of the equipment and estimated the values for the cost variables of the model.

For the purpose of this simulation, we took the approximate number of main transmitter sites of public service broadcaster in Serbia for the number of transmitter sites in our model $(t)$ which is $t=12$. Different national broadcasters in Serbia own different number of transmitters.

$\sum_{t=1}^{M} S U B_{t}$ is the sum of cumulative number of subscribers calculated by discrete Bass model using formulas (7) and (8), as explained earlier with coefficients $p=$ $0.030, q=0.060$ and the variation of $m, m=45,000$ and $m=75,000$.

Finally, $P$ is input variable which is not easy to estimate as it depends on indeed many different factors. We have estimated it for Serbian market taking into account the wholesale price that an efficient retailer could afford to pay (inexpensive content), reasonable local efficient retail costs, level of subscription for similar service (IPTV from incumbent carrier), without bundling to other services and the need to earn a return. The values that are included in this simulation for $P$ (profit from one PayTV program per subscriber per month) are $0.1 €$ and $0.2 €$.

For the Serbian digital terrestrial broadcasting project, the estimated number of TV programs for defined coverage zone $(n)$ is 33 and, by using diagram in Figure 3 and Equation (16), we calculated the number of TV programs per one multiplex in DVB-T $-j_{1}=9$, the number of TV programs per one multiplex in DVB-T2 $-j_{2}=16, k=4$ number of multiplexes and RF channels and $a=28$ number of TV programs. The simulation results from profit/loss analysis we have carried out by applying the model to the Serbian market are presentedon the four following diagrams. Except two input variables $(P$ and $m)$, all others are kept constant as defined in this section. The commercial parameter $P$ and market parameter $m$ are taken with two different values each.

In Figure 4, the diagram presents total cost $(T C)$ and total revenue $(T R)$ curve if $P=0.1$ and $m=45,000$, showing break-even point reached after approximately 48 months from the network and service launch date.

In Figure 5, the diagram presents total cost $(T C)$ and total revenue $(T R)$ curve if $P=0.1$ and $m=75,000$, showing break-even point reached after approximately 35 months from the network and service launch date. 


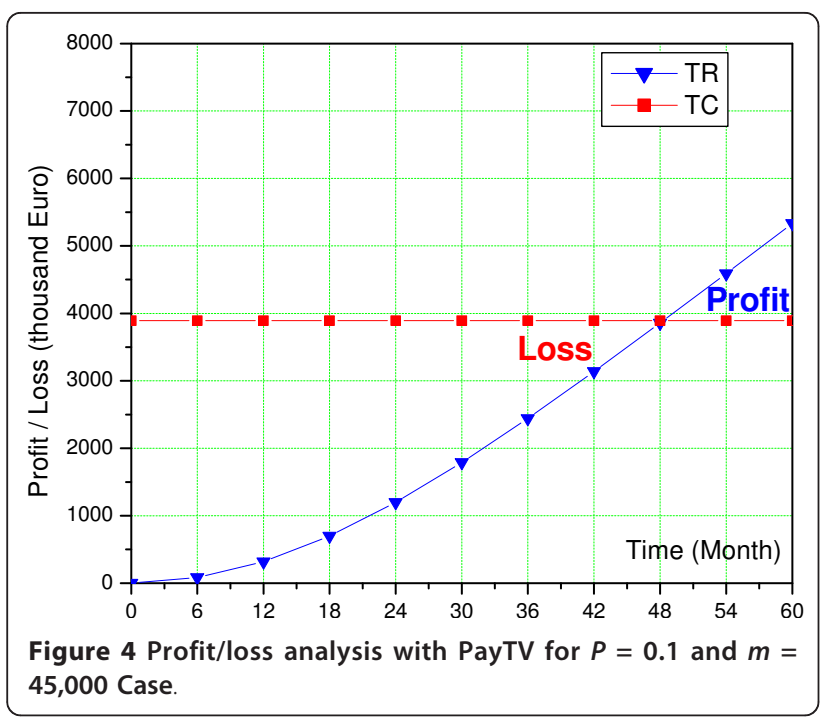

In Figure 6, the diagram presents total cost $(T C)$ and total revenue $(T R)$ curve if $P=0.2$ and $m=45,000$, showing break-even point reached after approximately 32 months from the network and service launch date.

In Figure 7, the diagram presents total cost $(T C)$ and total revenue $(T R)$ curve if $P=0.2$ and $m=75,000$, showing break-even point reached after approximately 24 months from the network and service launch date.

Analyzing the results of profit/loss calculations for Serbian Case, we can conclude that in order to fulfill the requirement for the number of TV programs for defined coverage $(n=33)$, the number of RF channels is kept the same $k=4$ for both standards (DVB-T/T2), and due to technology improvements DVB-T2 can support $a=28$ more PayTV programs than DVB-T. In that case the calculations performed by our model bring us to simulations results of reaching break-even point

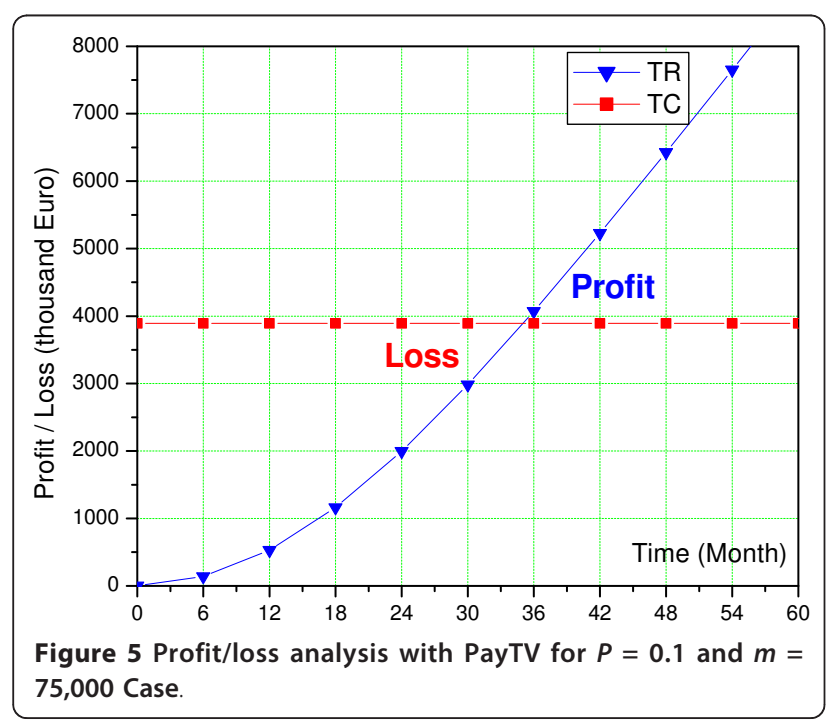

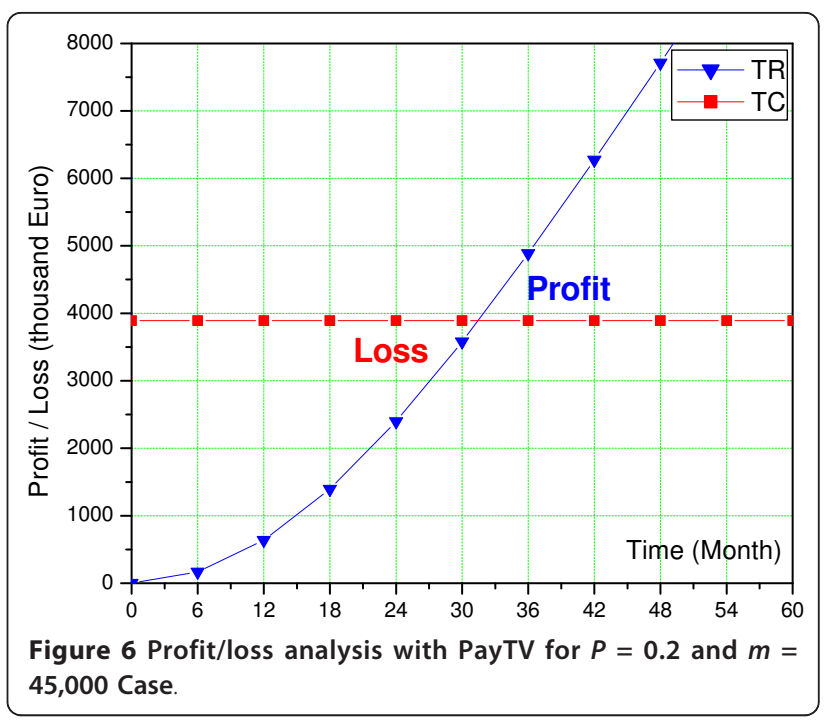

between 2 and 4 years after service launch, depending on values of $P$ and $m$ parameters chosen. The fastest profitability of the overall infrastructure investment is reached with $P=0.2$ and $m=75,000$ Case (2 years) and the slowest with $P=0.1$ and $m=45,000$ Case ( 4 years). As the model is nonlinear, it could be seen from diagrams that by doubling the PayTV profit $(P)$ per subscriber per program (the difference of retail and wholesale service price) we cannot cut the time to total profit by two. Depending on ultimate number of service subscribers, the time to total profit is shorten from 48 to 32 months for $m=45,000$ and the time to total profit is shorten from 35 to 24 months for $m=75,000$. On the other hand, if we cut almost by two the ultimate number of subscribers (from 75,000 to 45,000 ) the time to total profit is extended from 35 to 48 months for $P=$

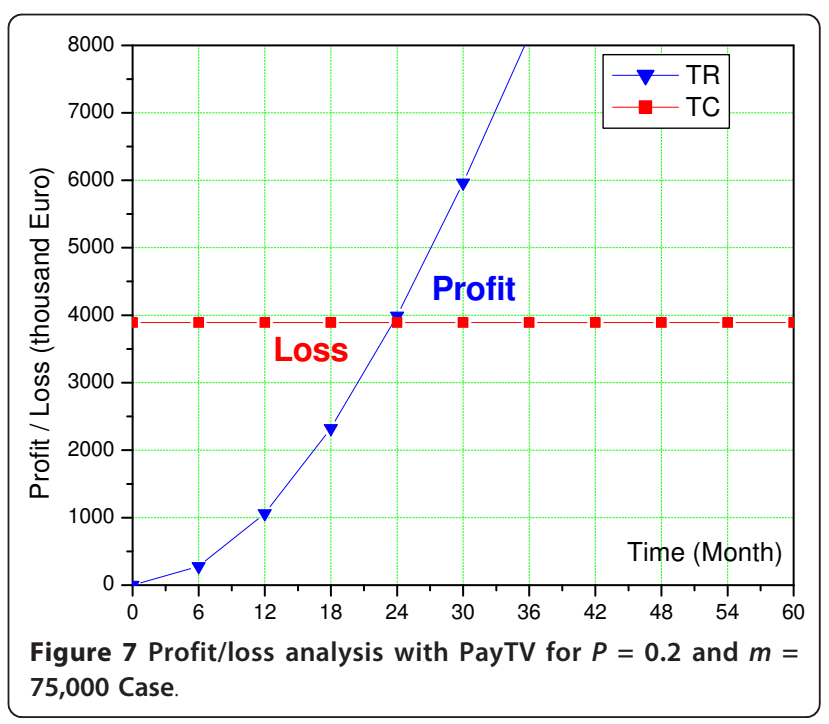


0.1 and the time to total profit is extended from 24 to 32 months for $P=0.2$.

Now we will show the simulation results for the model described in the previous section where the revenue is calculated from PayTV service and license for mobile broadband services issued in the spectrum auction process.

For the input variables, we have taken the same values as earlier in this section. The exception is variable $n$, which is not anymore input variable and it is calculated by using formula (18). In this variation of the model, the input variable is $k$ (the number of multiplexes) and it can take positive integer values. In this simulation for variable $k$, we took input values $4,5,6$, and 7 , which correspond to four different TR curves (later highlighted as $T R_{1}, T R_{2}, T R_{3}$, and $T R_{4}$ ). We took 4 and more as the number of multiplexes since, for the previous simulation, we made calculation with four multiplexes and in Serbia there is a trend of increasing number of multiplexes for national coverage. The calculation of $T C$ is done by using formulas (10), (11), and (12). The model then uses Equations (13), (15), and (18) for obtaining values for $j_{1}, j_{2}$, and $n$, respectively. In order to calculate the available spectrum for auction $S$, we then use formulas (19), (20), (21), and (22), and for calculating a number of PayTV programs Equation (24) is used. Now with formula (23) we calculate the first component of digital dividend, which comes from the revenue of MBA process. For that calculation we need two input values, $P S$ and $P O P$. Defining $P O P$ is much easier as this is statistical value and according to just finished census in Serbia there are around 7.2 million people living in the country (the process is not yet fully completed). Regarding the price for spectrum $P S$, we need to estimate that value, as in Serbia the frequency spectrum has not been sold on the auction, including the $800 \mathrm{MHz}$ band. After the analysis of data from [21], we estimated PS for Serbian $800 \mathrm{MHz}$ band as $0.1 € / \mathrm{MHz} /$ pop. It is a quite conservative estimation and we did not want, by taking a larger value, to increase unrealistically this component of revenue. For instance, the value of $0.1 € / \mathrm{MHz} /$ pop is about seven times lower than that achieved in Germany for $800 \mathrm{MHz}$ band on auction in May 2010 and at the same time it is in the range of price achieved again in Germany, but for $2 \mathrm{GHz}$ (it is normal that the prices paid for the higher bands are only a fraction of the 800 $\mathrm{MHz}$ band). We estimated conservative value achieved on auction also because we do not expect new mobile entrants in Serbia (only three existing operators bid for the spectrum) and because $800 \mathrm{MHz}$ spectrum would, most likely in the beginning, be used for rural areas where the business case is not so strong (the coverage and the capacity in urban areas at the moment are very good).
Finally, we calculated the total revenue with formula (25) where we used the same values of Bass parameters as in the previous simulation. The results are presented in Figures 8 (if $P=0.1$ and $m=45,000$ ) and 9 (if $P=$ 0.2 and $m=75,000)$. In each of these two diagrams, there are four curves for $T R$, where $T R_{1}$ corresponds to $k=4, T R_{2}$ corresponds to $k=5, T R_{3}$ corresponds to $k=$ 6, and $T R_{4}$ corresponds to $k=7$.

Figure 8 shows that the investment in DVB-T2 technology is returned immediately if the number of multiplexes is larger than 4 . In the case of $k=4$ and $n=36$, with DVB-T2 standard we obtain one spare UHF channel $(2 \times 2.5 \mathrm{MHz}$ for LTE $)$ and $12 \mathrm{TV}$ programs for PayTV service (curve $T R_{1}$ ), and the profitability is achieved after 18 months from the auction and PayTV service launch. In other three cases the profitability is achieved just after the spectrum auction is finished. The number of PayTV programs has the strongest influence on profit value over time. This is all happening if $P=$ 0.1 and $m=45,000$. If we change the ultimate number of PayTV service subscribers from $m=45,000$ to $m=$ 75,000 , and the profit from one PayTV program per subscriber per month changes from $P=0.1 €$ to $P=0.2$ $€$, we will obtain the simulation results as shown in Figure 9. The results are similar as in the previous figure but the revenue from PayTV service comes faster. Because of that, in the case of $k=4$ and $n=36$ (curve $T R_{1}$ ), the profitability is achieved after 9 months from the auction and PayTV service launch. In other three cases $\left(T R_{2}, T R_{3}\right.$, and $\left.T R_{4}\right)$ the profitability is achieved immediately after the spectrum auction is finished, but profit values in these scenarios increase faster than in Figure 8. If we are interested not only in break-even point, but also in profit value over time, for instance after 3 or 6 years, from Figure 9 we can see that after 6

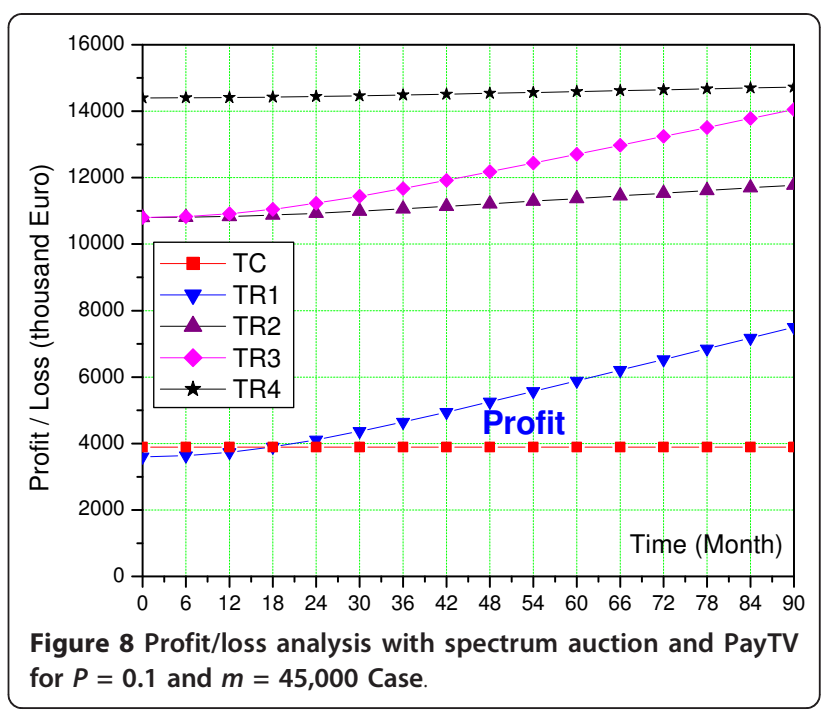




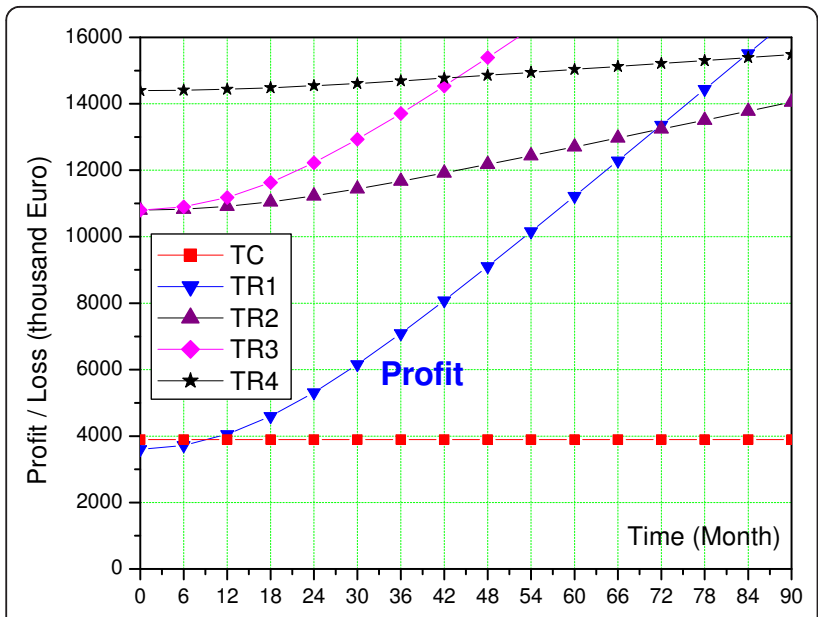

Figure 9 Profit/loss analysis with spectrum auction and PayTV for $P=0.2$ and $m=75,000$ Case.

years profit value for the case $T R_{1}$ will overcome profit value for the case $T R_{2}$ even though the initial revenue for the case $T R_{1}$ is three times lower than for the case $T R_{2}$. By choosing different technology profiles for DVB$\mathrm{T}$ and DVB-T2, MPEG-4 bit rate, as well as the number of multiplexes $k$, we can control the profitability over time (whether we want immediate profitability but with lower value over time or postponed profitability with a higher value at a later stage) which could be predicted with this simulation model.

Further to the discussion above, it seems that DVB-T2 technology improvements have a very strong influence on digital broadcasting business case, and if the digital broadcasting system is built on the analog broadcasting infrastructure base, the usage of DVB-T cannot be any more easily justified as DVB-T2 can return the investment in just a couple of years (Serbian case with revenue from only PayTV service), or immediately if the digital dividend spectrum is sold on the auction, depending on the market and commercial estimations/ circumstances. Naturally, the model could be applied to any other market/country but with different DVB-T/T2 technology profile chosen, Bass Diffusion Model parameters, and other technical, market and commercial values of the variables.

\section{Conclusion}

This article analyses the technical parameters for deploying the latest broadcast technologies and proposes a model that evaluates the economic efficiency of DVB$\mathrm{T}$ against DVB-T2. Our previous research caused a method of estimating the benefits of digital dividend which is in this article refined by comparing directly DVB-T2 and DVB-T, applying the model to multiple multiplex scenario, estimating PayTV service acceptance rate by Bass Diffusion Model and evaluating the breakeven point for the overall investment in DVB-T2 broadcasting infrastructure, based on the revenue from capacity gain (advanced digital dividend) due to DVB-T2 technology improvements. The proposed model is simple and flexible, but it is also very useful for estimating the efficiency of technology and for strategic decision making in the field of ubiquitous media enabled by digital broadcasting wireless network.

The model was applied to the Serbian broadcast market. The results from simulation show that if for the revenue we use only PayTV service, the DVB-T2 broadcast network profitability could be achieved in approximately 3 years if four multiplexes/RF channels are used, or even faster ( 2 years) -depending on the market situation. If for the revenue we use PayTV service and the spectrum auction at the same time, for more than four multiplexes the profitability is achieved immediately.

\section{Acknowledgements}

This study was partially supported by the Republic of Serbia, Ministry of Science and Technology Development, under the Grant No. 32048.

\section{Author details}

${ }^{1}$ ICT College of Applied Sciences, Zdravka Celara 16, 11000 Belgrade, Serbia ${ }^{2}$ Faculty of Electrical Engineering, University of Belgrade, Ministry of Telecommunications and Information Society, Bulevar kralja Aleksandara 73, Nemanjina 22-26, 11000 Belgrade, Serbia

\section{Competing interests}

The authors declare that they have no competing interests.

Received: 1 July 2011 Accepted: 18 February 2012 Published: 18 February 2012

\section{References}

1. W Fischer, Digital Video and Audio Broadcasting Technology, 3rd edn. Springer, Heidelberg, pp. 3-4. pp. 765-767 (2010)

2. A Sugaris, IReljin, Digital broadcasting techno-economic efficiency simulation model. Electron Electric Eng Mag. 17(3), 109-114 (2011)

3. TX Brown, DC Sicker, Examining the viability of broadband wireless access under alternative licensing models in the TV broadcast bands. EURASIP J Wirel Commun Netw. 2008 (2008). Article ID 470571, 12. doi:10.1155/2008/ 470571

4. J Kim, D Lee, J Ahn, D-S Ahn, B-J Ku, Is HAPS viable for the next-generation telecommunication platform in Korea?. EURASIP J Wirel Commun Netw. 2008 (2008). Article ID 596383, 8. doi:10.1155/2008/596383

5. R Tadayoni, KE Skouby, Terrestrial digital broadcasting: convergence and its regulatory implications. Telecommun Policy. 23(2), 175-199 (1999). doi:10.1016/S0308-5961(98)00086-X

6. A Hoikkanen, Economics of wireless broadcasting over DVB-H networks, in the 5thWireless Telecommunications Symposium (WTS'06), Pomana, CA, p. 5 (April 2006). doi:10.1109/WTS.2006.334521

7. D Skiöld, An economic analysis of DAB \& DVB-H. EBU Tech Rev. 2006(305), 10 (2006)

8. J Yip, Digital TV development: techno-economic planning considerations, in Digital TV Symposium, Session 9: Business Planning and Regulatory Issues, Asia-Pacific Broadcasting Union, Kuala Lumpur, Malaysia, (26-27 March 2007)

9. Analysys Mason, DotEcon and Hogan\&Hartson, Exploiting the digital dividend - a European approach. Report for the European Commission (2009)

10. WE Deming, Out of the Crisis, MIT Center for Advanced Engineering Study, Cambridge, MA, p. 88 (1986). ISBN 0-911379-01-0

11. S Verbrugge, K Casier, JV Ooteghem, B Lannoo, Practical steps in technoeconomic evaluation of network deployment planning, in White Paper, 
Ghent University, Department of Information Technology, INTEC Broadband Communication Networks http://www.ict-oase.eu/public/files/

IBCN_TE_WhitePaper_200911.pdf (19 November 2009). Accessed 16 June 2011

12. I Reljin, A Sugaris, DVB standards development, in Proceedings of the 9th International Conference on Telecommunications in Modern Satellite, Cable and Broadcasting Services (TELSIKS'09), Nis, Serbia, pp. 263-272 (October 2009)

13. ETSI European Standard (Telecommunications series), Digital Video Broadcasting (DVB), Frame structure channel coding and modulation for a second generation digital terrestrial television broadcasting system (DVBT2), ETSI EN 302755 V1.1.1. (September 2009)

14. DVB Blue Book, Implementation guidelines for a second generation digital terrestrial television broadcasting system (DVB-T2), DVB Document A133. (June 2010)

15. P Jallon, An Algorithm for Detection of DVB-T Signals Based on Their Second-Order Statistics. EURASIP J Wirel Commun Netw. 2008 (2008). Article ID 538236, 9. doi:10.1155/2008/538236

16. E Rogers, Diffusion of Innovations, (Free Press, Glencoe, 1962). ISBN0612628434

17. FM Bass, A new product growth for model consumer durables. Manag Sci. 15(5), 215-227 (1969). doi:10.1287/mnsc.15.5.215

18. V Srinivasan, C Mason, Nonlinear least squares estimation of new product diffusion models. Market Sci. 5(2), 169-178 (1986). doi:10.1287/mksc.5.2.169

19. FM Bass, K Gordon, T Ferguson, M Githens, DIRECTV: forecasting diffusion of a new technology prior to product launch. INTERFACES. 31(3), S82-S93 (2001). doi:10.1287/inte.31.3s.82.9677

20. G Lilien, A Rangaswamy, Marketing Engineering: Computer-Assisted Marketing Analysis and Planning, 2nd edn. (Trafford Publishing, Canada, 2004), p. 259. revised

21. S Zehle, G Friend, Twice-shy network operators reject high spectrum prices. News Analysis http://www.computerweekly.com/Articles/2010/09/23/ 243013/Twice-shy-network-operators-reject-high-spectrum-prices.htm (23 September 2010). Accessed 02 November 2011

doi:10.1186/1687-1499-2012-52

Cite this article as: Sugaris and Reljin: DVB-T2 technology improvements challenge current strategic planning of ubiquitous media networks. EURASIP Journal on Wireless Communications and Networking 2012 2012:52.

\section{Submit your manuscript to a SpringerOpen ${ }^{\mathcal{O}}$ journal and benefit from:}

- Convenient online submission

- Rigorous peer review

- Immediate publication on acceptance

- Open access: articles freely available online

- High visibility within the field

- Retaining the copyright to your article

Submit your next manuscript at $\gg$ springeropen.com 